J. Appl. Cryst. (1975). 8, 184

Pseudopotential Calculations of Lattice Distortions around Impurities in Simple Metals

\author{
By A. BaratofF \\ IBM Zürich Research Laboratory* and Kernforschungsanlage Jülich \\ AND E. SEITZ \\ Institut für Festkörperforschung, Kernforschungsanlage Jülich, Postfach 365, 517 Jülich, Germany (BRD)
}

(Received 29 April 1974)

\begin{abstract}
Second-order pseudopotential calculations are known to reproduce fairly well the structure and phonon spectra of many simple metals (without $d$-states close to the Fermi level) [Heine, V. \& Weaire, D. (1971). Solid State Physics, 24, 249-463]. The underlying assumption of linear screening should also be reasonable for impurities with valences and core radii close to those of the host. Its validity can be checked a posteriori and by comparing calculated properties with measurements on dilute alloys (lattice expansion, heat of solution, electron scattering lifetime) [Benedek, R. \& Baratoff, A. (1973). Solid State Commun. 13, 385-388] and, especially, coherent elastic neutron scattering [Schumacher, H., Schmatz, W. \& Seitz, E. (1973). Phys. Stat. Sol. (a), 20, 109-117]. The resulting description in terms of pairwise screened hosthost and impurity-host interactions, in conjunction with the lattice-statics approximation [Matsubara, T. J. (1952). J. Phys. Soc. Japan, 7, 270], enables one to carry out a self-consistent calculation of the static structure factor $S(q)$ to first order in the displacements. The calculations to be reported on alkalis in alkalis and on impurities in aluminum are based on Shaw's optimized model potential and on a screening approximation satisfying the compressibility sum rule [Shaw, R. W. (1968). Phys. Rev. 174, 769-781; (1969). J. Phys. C: Solid State Phys. 2, 2335-2449; (1970). J. Phys. C: Solid State Phys. 3, 1140-1158; Vashishta, P. \& Singwi, K. S. (1972). Phys. Rev. B6, 875-887.] Special care has been taken to ensare proper convergence, a problem neglected in previous similar calculations. Calculated structure factors exhibit features associated with appreciable interactions beyond nearest neighbours of the defect and/or with incipient structural instabilities of the host lattice.
\end{abstract}

\footnotetext{
* Present address.
}

J. Appl. Cryst. (1975). 8, 184

\title{
Lattice Distortions by Copper Atoms in an Aluminum Lattice
}

\author{
By G. Bauer ANd E. Seitz \\ Institut für Festkörperforschung der Kernforschungsanlage Jülich, Postfach 365, 517 Jülich, Germany (BRD)
}

(Received 29 April 1974)

With the method of elastic diffuse scattering of neutrons the strain field produced by copper atoms dissolved in aluminum was investigated. Measurements were performed at $800 \mathrm{~K}$ and at room temperature on a single crystal containing 0.8 at. $\%$ of copper. A time-of-flight technique was applied to discriminate between elastically and inelastically scattered neutrons. The general features of the scattering pattern recorded in the high-temperature run are found to be well explained by a theoretical approach based on the assumption of isotropi: forces acting on nearest neighbours only. Possible refinements of the model are discussed. At room temperature the pattern shows the characteristic features resulting from the formation of Guinier-Preston zones. 